\title{
INTERVENCIONES PSICOLÓGICAS PARA EL MANEJO Y REDUCCIÓN DE ESTRÉS EN PACIENTES HIPERTENSOS: UNA REVISIÓN SOBRE SU EFECTIVIDAD
}

\author{
PSYCHOLOGICAL INTERVENTIONS ON STRESS MANAGEMENT AND REDUCTION \\ FOR HYPERTENSIVE PATIENTS: A REVIEW OF THEIR EFFECTIVENESS
}

\author{
ITA HORTENCIA FlORES-VALDEZ*, MARTHA PATRICIA LEÓN-SANTOS**, ELVIRA VERA-HERNÁNDEZ**** \\ Y MARÍA DEL ROCÍO HERNÁNDEZ-POZO***** \\ UNIVERSIDAD NACIONAL AUTÓNOMA DE MÉXICO - MÉXICO
}

FECHA RECEPCIÓN: 21/6/2013・ FECHA ACEPTACIÓN: 3/9/2013

Para citar este artículo: Flores-Valdez, I., León-Santos, M., Vera-Hernández, E., \& Hernández-Pozo, M. (2013). Intervenciones psicológicas para el manejo y reducción de estrés en pacientes hipertensos: una revisión sobre su efectividad. Psychologia: avances de la disciplina, 7(2), 25-44.

\begin{abstract}
Resumen
La hipertensión está considerada como una de las principales problemáticas de salud pública, pues es una enfermedad crónica asintomática caracterizada por una elevación de presión arterial, en la que inciden los niveles de estrés que percibe el paciente y cuyas consecuencias pueden ser devastadoras tanto en la salud física como emocional de este grupo. Se realizó una revisión analítica enfocada a la efectividad de diversos tipos de intervención psicológica, para disminuir los niveles de presión sistólica, controlar el nivel de la presión diastólica, disminuir la percepción del estrés de los sujetos y propiciar una mayor adherencia al tratamiento. La búsqueda se realizó en la base de datos Springer, donde 50 artículos cumplieron con los criterios de exclusión y de inclusión, para el periodo 2000-2013. La revisión arrojó una mayor inclinación hacia las intervenciones de tipo conductual (38\%), sin embargo, las intervenciones cognitivo-conductuales fueron las más eficaces para disminuir los niveles de estrés y generar la reducción más significativa en presión arterial, con un 100\% de resultados positivos, mientras que solo el 68.4\% de los estudios conductuales reportó una mejoría.

Palabras clave: Hipertensión, estrés, tratamiento, conductual, cognitivo, técnicas.
\end{abstract}

* Este estudio se realizó durante el seminario que cursaron las primeras tres autoras bajo la supervisión de la cuarta autora, como parte del programa de Licenciatura a Distancia en Psicología, SUAyED de la UNAM, FES Iztacala. Agradecemos las observaciones de los revisores anónimos que permitieron mejorar el escrito

** Universidad Nacional Autónoma de México, Facultad de Estudios Superiores Iztacala, Carrera de Psicología, Programa SUAyED, Tlalnepantla, Estado de México, México

*** Universidad Nacional Autónoma de México, Centro Regional de Investigaciones Multidisciplinarias, Programa de Equidad y Género, Cuernavaca, Morelos, México

****Universidad Nacional Autónoma de México, Facultad de Estudios Superiores Iztacala, Proyecto de Investigación en Aprendizaje Humano, Tlalnepantla, Estado de México, México. Dirigir la correspondencia a María del Rocío Hernández Pozo, CRIM-UNAM. Proyecto de Investigación Equidad y Género, Ave. Universidad s/n, Campus Ciudad Universitaria UAEM, Circuito 2, Col. Chamilpa, Cuernavaca, Morelos, C. P. 62210, México, correo electrónico: herpoz@unam.mx 


\begin{abstract}
Hypertension an asymptomatic chronic disease characterized by elevated blood pressure, is considered a public health problem, affected by stress levels perceived by the patient, with physical and emotional health consequences that can be devastating for this population. This study presents an analytical review focused on the effectiveness of different types of psychological intervention to reduce systolic blood pressure levels, controlling the level of diastolic pressure, decreasing stress perception of subjects and fostering greater adherence. The search was conducted by means of the Springer database, with 50 articles selected on the basis of the inclusion and exclusion criteria for the period 2000-2013. Results showed greater inclination towards behavioral type interventions (38\%), while cognitive-behavioral interventions were the most effective for decreasing stress levels and producing the most significant reduction in blood pressure, 100\% of the studies based on this type of intervention showed positive results, while the behavioral type only registered $68.4 \%$ of success.

Keywords: Hypertension, stress, treatment, behavioral, cognitive, techniques.
\end{abstract}

\section{Introducción}

El estrés es una sensación de tensión física y psicológica que suele ocurrir en situaciones percibidas por los individuos como difíciles o inmanejables, dependiendo de factores tales como la edad o el nivel sociocultural. González (2006) señala al estrés como un estado psicofísico que se presenta cuando la demanda percibida por una persona excede la percepción de su capacidad para afrontarla.

De acuerdo a la Asociación Americana de Psicología (2013) niveles elevados de estrés que comúnmente aumentan la probabilidad de un comportamiento hostil, pueden empeorar factores de riesgo cardiovascular existentes y producir enfermedades del corazón.

Tomando en cuenta lo anterior, Snieder et ál. (2002) y Treiber, Davis y Turner (2001), indican que «se ha considerado que el estrés ambiental y psicosocial han desempeñado un papel significativo en el desarrollo de la hipertensión mediante su actuación en los mecanismos de control agudo y a largo plazo de la presión arterial» (Barnes \& Orme, 2008, p. 2).

Guyton y Hall (1996; citado en Barnes \& Orme, 2008, pp. 2-3) señalan que «la exposición al estrés trae como resultado aumentos en la presión arterial (PA) mediados por el Sistema Nervioso Simpático (SNS) para satisfacer la necesidad inmediata de un mayor riego sanguíneo al cerebro y al cuerpo. La activación del SNS a su vez acelera el sistema renina-angiotensina-aldosterona (RAAS), que incluye la liberación de la hormona vasoconstrictora angiotensina II, que contribuye al aumento agudo de la presión arterial». Por su parte, Snieder et ál. (2002) y Treiber, Davis y Turner
(2001), indican que «este patrón de respuesta se asocia con aumentos concomitantes de la tensión cardíaca, de la pared vascular y del estrés intravascular de cizallamiento (fuerza aplicada o presión ejercida contra la superficie y las capas de la piel a medida que los tejidos se deslizan en planos opuestos, pero paralelos) que a lo largo del tiempo contribuye a la remodelación vascular y ventricular precoz que finalmente conducen a la hipertensión» (Barnes \& Orme, 2008, pp. 2-3).

Una vez expuesto lo concerniente al estrés, cabe señalar la importancia del manejo del mismo, pues permite que el individuo logre controlar y reducir la tensión que surge cuando una determinada situación se percibe como difícil o inmanejable, a fin de contrarrestar los niveles negativos que el estrés tiene en su salud física, en este caso, la hipertensión. Para ello es necesaria la aplicación de diversas intervenciones.

En 1980 el Comité Nacional Conjunto para la Detección, Evaluación y Tratamiento de la Hipertensión en EE. UU., se manifestó oficialmente sobre la eficacia de algunas de las intervenciones conductuales más empleadas para combatir la hipertensión (retroalimentación biológica, psicoterapia y relajación), desestimando su uso para casos de hipertensión crónica, pues daban lugar a reducciones modestas que sólo se producían en algunos pacientes. El mismo comité cuatro años más tarde subrayó los beneficios de relajación y la retroalimentación biológica para casos de hipertensión leve, debido a que producían reducciones pequeñas, pero consistentes y significativas, recomendando el uso de ambas técnicas en conjunción con la administración de fármacos para casos severos de hipertensión. Más recientemente, en 1993 el mismo organismo subrayó la importancia de los aspectos 
conductuales y aconsejó los cambios en el estilo de vida para un tratamiento exitoso contra la hipertensión arterial (García, Labrador \& Sanz, 1999; citados en Molerio, Arce, Otero \& Nieves, 2005).

De igual manera y «con el fin de complementar los tratamientos llevados a cabo de forma exclusiva a partir de las técnicas conductuales aisladas, surgen los programas o paquetes de intervención cognitivo-conductual, los cuales combinan todo un conjunto de estrategias dirigidas a instruir al paciente en la gestión de las variables emocionales que influyen en la HTA (Hipertensión Arterial) y para dotarle de estrategias que le permitan mantener diferentes hábitos saludables para sus niveles de PA (Presión Arterial)». «Este tipo de programas de intervención se han aplicado al tratamiento de diferentes enfermedades de tipo coronario entre las que se incluye la HTA esencial mostrando una eficacia elevada» (Fernández, 2009, pp. 163-165).

Finalmente, tomando en cuenta la importancia que tiene el manejo y reducción de estrés para la hipertensión, así como los antecedentes de las intervenciones ya mencionadas, se realizó una revisión de artículos con el objetivo de identificar el tipo de intervenciones que se han empleado con mayor frecuencia para reducir la hipertensión arterial, así como para hacer un análisis comparativo de los resultados que han tenido las aplicaciones de esos métodos en años recientes.

\section{Método}

Para la selección de los artículos relativos al manejo y reducción de estrés asociado a hipertensión se consultó la base de datos para publicaciones científicas de la casa editorial Springer-Verlag, para el período comprendido de enero del 2000 a mayo del 2013. La estrategia de búsqueda consistió en identificar todos los artículos que en el título, resumen o palabras clave, tuvieran las frases descriptoras en inglés: stress reduction, behavioral intervention y/o hypertension.

A partir de los resultados de esa búsqueda, se seleccionaron artículos que adicionalmente cumplieran con alguno de los siguientes criterios: 1) mención de técnicas psicológicas en relación con las enfermedades cerebro-vasculares, 2) abordar la relación del estrés con enfermedades crónicas cardiovasculares, principalmente hipertensión, 3) mención sobre intervenciones psicológicas frente a las enfermedades cerebro-vasculares, 4) mención de algunos de los factores de riesgo asociados a la hipertensión y 5) que fueran artículos originalmente escritos en inglés o español, disponibles en su versión completa a través de la base Springer-Verlag.

Finalmente se excluyeron los trabajos realizados con animales y estudios que solo se referían a la validación de algún instrumento psicométrico.

\section{Resultados}

La búsqueda a partir de los términos señalados arrojó inicialmente 75 artículos, de los cuales 25 no cumplían todos los criterios de inclusión, pues la mayoría de ellos eran de publicaciones previas a 2000, también se eliminaron estudios que trataban exclusivamente con validaciones de instrumentos psicométricos. Finalmente de acuerdo a los criterios de inclusión, se seleccionó un total de 50 artículos, todos ellos relacionados con el manejo y la reducción de estrés asociados a la hipertensión, los cuales fueron publicados en Estados Unidos, Canadá, Suecia, Italia, Reino Unido, Portugal y Australia, durante el periodo comprendido del 2000 al 2013.

Analizando la distribución geográfica de los escritos quedó en evidencia un gran interés sobre el tema en Estados Unidos ya que se identificaron 36 artículos publicados con ese origen, mientras que en ningún otro país se publicaron más de cuatro artículos durante ese periodo, por ejemplo en Canadá y Suecia se publicaron cuatro, en Italia e Inglaterra dos y en Portugal y Australia uno.

Por otro lado, la mayor frecuencia de artículos se registró en los años 2011 y 2012, siendo muy baja la producción de artículos sobre el tema en los años 2000, 2002, 2004 y 2005, como se puede apreciar en la figura 1. Es muy probable que la tendencia al alza en la publicación sobre intervenciones relacionadas con la disminución del estrés orientada a la población hipertensa continúe, ya que en lo que va del año del 2013, hasta el mes de mayo van cinco artículos publicados con esa temática. 


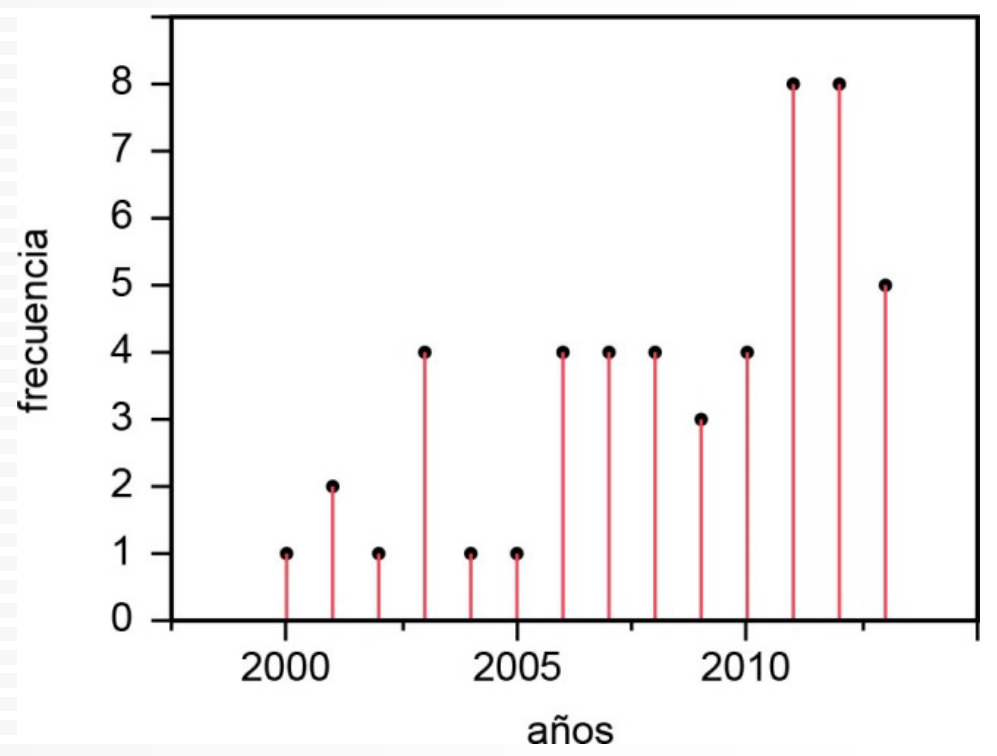

Figura 1. Distribución de artículos sobre intervenciones psicológicas contra la hipertensión en la base bibliométrica Springer-Verlag

En la tabla 1 se condensan los tipos de intervenciones abordadas por los artículos seleccionados, así como el tamaño de la muestra y sus características, identificando a los escritos por su primer autor, el año de publicación y el país de origen. Esos artículos que constituyen el universo de análisis del estudio, se identifican en la lista de referencias mediante un asterisco, como es costumbre en los estudios de revisión analítica.

Tabla 1. Estudios dirigidos al manejo y control del estrés asociado a la hipertensión, en función del tipo de intervenciones y población participante.

\begin{tabular}{|c|c|c|c|c|c|}
\hline $\begin{array}{l}\text { Año de } \\
\text { publicación }\end{array}$ & Primer autor & $\begin{array}{l}\text { País de } \\
\text { publicación }\end{array}$ & $\begin{array}{c}\text { Tipo de } \\
\text { intervención }\end{array}$ & $\mathrm{N}$ & Características (Muestra) \\
\hline 2000 & Winefield, H. & EE. UU. & Conductual & 291 & $\begin{array}{l}\text { Hombres y mujeres cuidadores de } \\
\text { pacientes psiquiátricos }\end{array}$ \\
\hline 2001 & Clark, R. & EE. UU. & Conductual & 39 & $\begin{array}{l}\text { Mujeres afroamericanas estudiantes de } \\
\text { posgrado }\end{array}$ \\
\hline 2001 & Munz, D. & EE. UU. & Cognitiva & 300 & $\begin{array}{l}\text { Empleados de servicio al cliente y de } \\
\text { ventas de una empresa }\end{array}$ \\
\hline 2002 & Cruess, S. & EE. UU. & Conductual & 100 & Hombres de 23 a 52 años de edad. \\
\hline 2003 & Barnes, V. & EE. UU. & Conductual & 45 & $\begin{array}{l}\text { Adolescentes afroamericanas } \\
\text { hipertensas de } 15 \text { a } 18 \text { años. }\end{array}$ \\
\hline 2003 & Overhaus, S & EE. UU. & Conductual & 6 & $\begin{array}{l}\text { Con hipertensión leve y } 5 \text { normotensos } \\
\text { de } 25 \text { a } 59 \text { años de edad. }\end{array}$ \\
\hline 2003 & Sudin, Ö. & Suecia & Multifactorial & 132 & Hombres con enfermedades coronarias. \\
\hline 2003 & Vermunt, $\mathrm{R}$ & EE. UU. & Conductual & 80 & $\begin{array}{l}34 \text { hombres y } 46 \text { mujeres de } 17 \text { a } 25 \\
\text { años con hipertensión }\end{array}$ \\
\hline 2004 & Vocks, S. & EE. UU. & $\begin{array}{l}\text { Cognitivo- } \\
\text { conductual }\end{array}$ & 101 & $\begin{array}{l}\text { Pacientes de un centro de rehabilitación } \\
\text { psicosomática, en edad de entre } 20 \text { y } \\
55 \text { años. }\end{array}$ \\
\hline
\end{tabular}

Esta tabla continúa en la siguiente página $\longrightarrow$ 


\begin{tabular}{|c|c|c|c|c|c|}
\hline $\begin{array}{c}\text { Año de } \\
\text { publicación }\end{array}$ & Primer autor & $\begin{array}{c}\text { País de } \\
\text { publicación }\end{array}$ & $\begin{array}{c}\text { Tipo de } \\
\text { intervención }\end{array}$ & $\mathrm{N}$ & Características (Muestra) \\
\hline 2005 & Rhenen, W. & EE. UU. & $\begin{array}{l}\text { Cognitivo- } \\
\text { conductual }\end{array}$ & 130 & $\begin{array}{l}\text { Hombres y mujeres con edades entre } \\
18 \text { y } 63 \text { años. }\end{array}$ \\
\hline 2006 & Penedo, F. & EE. UU. & $\begin{array}{l}\text { Cognitivo- } \\
\text { conductual }\end{array}$ & 191 & $\begin{array}{l}\text { Hombres con una media de edad de } \\
65.1 \text {, con cáncer de próstata en etapa } \\
\text { I o II. }\end{array}$ \\
\hline 2006 & Shimazu, A. & EE. UU. & Conductual & 300 & Empleados de una empresa japonesa. \\
\hline 2006 & Reyes, G. & EE. UU. & $\begin{array}{l}\text { Cognitivo- } \\
\text { conductual }\end{array}$ & 10 & $\begin{array}{l}\text { Pacientes hipertensos leves primarios y } \\
\text { normotensos. }\end{array}$ \\
\hline 2006 & Carrico, A. & EE. UU. & Conductual & 130 & Hombres homosexuales y bisexuales. \\
\hline 2007 & Ben, D. & Portugal & $\begin{array}{l}\text { Prevención } \\
\text { primaria }\end{array}$ & $\mathrm{NE}$ & Adultos mayores \\
\hline 2007 & Daubenmier, J. & E.E.U.U. & $\begin{array}{l}\text { Prevención } \\
\text { primaria y } \\
\text { secundaria }\end{array}$ & 869 & $\begin{array}{l}293 \text { mujeres y } 576 \text { hombres con } \\
\text { enfermedad coronaria. }\end{array}$ \\
\hline 2007 & Han, H.R. & EE. UU. & Preventiva & 445 & $\begin{array}{l}\text { Pacientes coreanos, de } 40 \text { a } 64 \text { años de } \\
\text { edad }\end{array}$ \\
\hline 2007 & Townsend, T. & EE. UU. & Cognitiva & 33 & Mujeres afroamericanas \\
\hline 2008 & Carmody, J. & EE. UU. & $\begin{array}{l}\text { Cognitivo- } \\
\text { conductual }\end{array}$ & 174 & $\begin{array}{l}\text { Adultos, hombres y mujeres inscritos } \\
\text { en la Universidad de Massachusetts, } \\
\text { con enfermedades relacionadas con el } \\
\text { estrés. }\end{array}$ \\
\hline 2008 & Hekcler, E.B. & EE. UU. & $\begin{array}{l}\text { Cognitivo- } \\
\text { conductual }\end{array}$ & 102 & $\begin{array}{l}\text { Personas con hipertensión, de } \\
\text { nacionalidad afroamericana }\end{array}$ \\
\hline 2008 & Rausch, S. & EE. UU. & Conductual & 313 & $\begin{array}{l}20 \text { hombres y } 91 \text { mujeres } \\
\text { afroamericanas; y } 64 \text { hombres y } 138 \\
\text { mujeres europeos. }\end{array}$ \\
\hline 2008 & Scala, D. & Italia & Conductual & 292 & Hipertensos de origen italiano. \\
\hline 2009 & Beckett, N. & $\begin{array}{l}\text { Reino } \\
\text { Unido }\end{array}$ & $\begin{array}{l}\text { No } \\
\text { farmacológica y } \\
\text { farmacológica }\end{array}$ & $\mathrm{NE}$ & Personas mayores de 80 años \\
\hline 2009 & Blom, M. & Suecia & Conductual & 247 & $\begin{array}{l}\text { Mujeres de entre } 35 \text { y } 75 \text { años, } \\
\text { pacientes del Hospital de la Universidad } \\
\text { Karolinska, Huddinge. }\end{array}$ \\
\hline 2009 & McCraty, R. & EE. UU. & Promocional & 75 & Oficiales correccionales \\
\hline 2010 & Gold, E. & $\begin{array}{l}\text { Reino } \\
\text { Unido }\end{array}$ & $\begin{array}{l}\text { Cognitivo- } \\
\text { conductual }\end{array}$ & 9 & $\begin{array}{l}\text { Profesores de primaria y auxiliares } \\
\text { docentes. }\end{array}$ \\
\hline \multirow[t]{2}{*}{2010} & Labelle, L.E. & EE. UU. & Conductual & $\mathrm{NE}$ & $\begin{array}{l}\text { Reclutados de la lista de espera para } \\
\text { MBSR en Calgary, Canadá. }\end{array}$ \\
\hline & & & & & Esta tabla continúa en la siguiente página $\longrightarrow$ \\
\hline
\end{tabular}




\begin{tabular}{|c|c|c|c|c|c|}
\hline $\begin{array}{l}\text { Año de } \\
\text { publicación }\end{array}$ & Primer autor & $\begin{array}{l}\text { País de } \\
\text { publicación }\end{array}$ & $\begin{array}{c}\text { Tipo de } \\
\text { intervención }\end{array}$ & $\mathrm{N}$ & Características (Muestra) \\
\hline 2010 & Olsson, E. & Suecia & Conductual & 19 & $\begin{array}{l}\text { Personas con hipertensión, en edades } \\
\text { de entre } 25 \text { y } 75 \text { años. }\end{array}$ \\
\hline 2010 & Stauder, A. & EE. UU. & Conductual & 107 & $\begin{array}{l}\text { Hombres y mujeres entre } 18 \text { y } 74 \text { años } \\
\text { de edad. Con un grado mínimo de } \\
\text { educación a nivel secundaria. }\end{array}$ \\
\hline 2011 & Campbell, T. & Canadá & $\begin{array}{l}\text { Cognitivo- } \\
\text { conductual }\end{array}$ & $\mathrm{NE}$ & $\begin{array}{l}\text { Mujeres mayores de } 18 \text { años con } \\
\text { diagnóstico de cáncer y presión arterial. }\end{array}$ \\
\hline 2011 & Chow, Y.W. & EE. UU. & Conductual & 132 & $\begin{array}{l}89 \text { mujeres y } 43 \text { hombres, con más de } \\
18 \text { años de edad. }\end{array}$ \\
\hline 2011 & $\begin{array}{l}\text { Epstein- } \\
\text { Lubow, G. }\end{array}$ & EE. UU. & Conductual & 9 & Mujeres de 48 a 73 años de edad \\
\hline 2011 & Fernández, S. & EE. UU. & $\begin{array}{l}\text { Multinivel } \\
\text { basada en la } \\
\text { evidencia }\end{array}$ & 1039 & $\begin{array}{l}\text { Afroamericanos hipertensos ambos } \\
\text { sexos, atendidos en } 30 \text { centros de salud } \\
\text { comunitarios del área metropolitana de } \\
\text { New York. }\end{array}$ \\
\hline 2011 & Lubow, G. & EE. UU. & Cognitiva & 9 & $\begin{array}{l}\text { Mujeres de } 48 \text { a } 73 \text { años cuidadoras de } \\
\text { ancianos. }\end{array}$ \\
\hline 2011 & Palomba, D. & Italia & Conductual & 24 & $\begin{array}{l}\text { Pacientes ambulatorios no medicados } \\
\text { con prehipertensión en fase } 1\end{array}$ \\
\hline 2011 & Phillips, K. & EE. UU. & $\begin{array}{l}\text { Cognitivo- } \\
\text { conductual }\end{array}$ & 128 & $\begin{array}{l}\text { Mujeres con cáncer de mama no } \\
\text { metastásico. }\end{array}$ \\
\hline 2011 & $\begin{array}{l}\text { Warren- } \\
\text { Findlow, J. }\end{array}$ & EE. UU. & Conductual & 190 & $\begin{array}{l}\text { Afroamericanos de por lo menos } 21 \\
\text { años. }\end{array}$ \\
\hline 2012 & Aroian, K. & EE. UU. & Preventiva & 17 & $\begin{array}{l}\text { Ocho profesionistas y nueve } \\
\text { trabajadores de servicio de alimentos, } \\
\text { de origen hispano. }\end{array}$ \\
\hline 2012 & Gayner, B. & Canadá & $\begin{array}{l}\text { Cognitivo- } \\
\text { conductual }\end{array}$ & 117 & Hombres homosexuales con VIH \\
\hline 2012 & $\begin{array}{l}\text { Orth-Gomér, } \\
\text { K. }\end{array}$ & Suecia & Conductual & .30 & $\begin{array}{l}11 \text { mujeres y } 19 \text { hombres hospitalizados } \\
\text { con síndrome coronario agudo. }\end{array}$ \\
\hline 2012 & Morone, N. & EE. UU. & $\begin{array}{l}\text { Cognitivo- } \\
\text { conductual }\end{array}$ & 74 & Adultos de Pittsburgh \\
\hline 2012 & Reginald, D. & Australia & $\begin{array}{l}\text { Cognitivo- } \\
\text { conductual }\end{array}$ & 33 & $\begin{array}{l}\text { Niños y jóvenes de } 7 \text { a } 17 \text { años de edad, } \\
\text { con trastorno de estrés postraumático }\end{array}$ \\
\hline 2012 & Zernicke, K. & Canadá & Multifactorial & 90 & $\begin{array}{l}\text { Pacientes tratados con síndrome de } \\
\text { intestino irritable }\end{array}$ \\
\hline 2012 & Walter, K. & EE. UU. & Cognitiva & 166 & $\begin{array}{l}\text { Hombres y mujeres veteranos con } \\
\text { trastorno de estrés postraumático con } \\
\text { una media de edad de } 51 \text { años. }\end{array}$ \\
\hline 2012 & $\begin{array}{c}\text { Van der } \\
\text { Weijer- } \\
\text { Bergsma, E. }\end{array}$ & EE. UU. & Preventiva & $\mathrm{NE}$ & $\begin{array}{l}\text { Niños de entre } 8 \text { y } 12 \text { años, de } 3 \\
\text { escuelas primarias en Ámsterdam, } \\
\text { Holanda }\end{array}$ \\
\hline
\end{tabular}

Esta tabla continúa en la siguiente página $\longrightarrow$ 


\begin{tabular}{|c|c|c|c|c|c|}
\hline $\begin{array}{l}\text { Año de } \\
\text { publicación }\end{array}$ & Primer autor & $\begin{array}{l}\text { País de } \\
\text { publicación }\end{array}$ & $\begin{array}{c}\text { Tipo de } \\
\text { intervención }\end{array}$ & $\mathrm{N}$ & Características (Muestra) \\
\hline 2013 & Bögels, S.M. & EE. UU. & Cognitiva & 86 & $\begin{array}{l}77 \text { mujeres y } 9 \text { hombres, con diversas } \\
\text { nacionalidades }\end{array}$ \\
\hline 2013 & Gans, J. & EE. UU. & $\begin{array}{l}\text { Cognitivo- } \\
\text { conductual }\end{array}$ & 514 & $\begin{array}{l}\text { Hombres y mujeres, de } 40 \text { a } 64 \text { años de } \\
\text { edad, coreo-americanos }\end{array}$ \\
\hline 2013 & Goyer, L. & Canadá & $\begin{array}{l}\text { Prevención } \\
\text { primaria }\end{array}$ & 185 & $\begin{array}{l}\text { Pacientes en situación de riesgo de } \\
\text { enfermedad cardiovascular }\end{array}$ \\
\hline 2013 & Mansyur, C. & EE. UU. & $\begin{array}{l}\text { Secuencial y } \\
\text { simultánea }\end{array}$ & 185 & $\begin{array}{l}65 \text { hombres y } 120 \text { mujeres } \\
\text { afroamericanos con hipertensión, entre } \\
45 \text { y } 65 \text { años de edad. }\end{array}$ \\
\hline 2013 & Anshel, M. & EE. UU. & Preventiva & 9 & $\begin{array}{l}\text { Ocho mujeres y un hombre, con edades } \\
\text { entre } 24 \text { a } 45 \text { años. }\end{array}$ \\
\hline
\end{tabular}

Nota. La palabra NE, hace referencia a que en el artículo examinado no se especificó el número de participantes.

Ahora bien, el número de participantes por estudio fue muy variado, es decir, en cinco no se especificó la cantidad total de personas que formaron parte del estudio, mientras que en los demás el número osciló entre seis y más de mil, el mayor número de participantes se registró en una investigación con 1039 afroamericanos neoyorquinos (Fernández, Tobin, Cassells, Díaz, Kalida \& Ogedegbe, 2011). De igual manera se detectó que el $6 \%$ de los estudios estuvo dirigido a hombres exclusivamente, el 16\% a las mujeres exclusivamente y el 78\% a ambos sexos, con edades de 7 a 80 años. Al realizar una comparación entre la proporción relativa de atención por sexo a los que se enfocaron las intervenciones para el control y reducción de estrés se pudo deducir que estas, han sido aplicadas sin preferencia de género, pues los estudios revisados muestran evidencia de que el interés se ha distribuido en ambos sexos equitativamente.

Por otro lado, el 4\% de los estudios se realizó con personas de orientación sexual atípica. También se registraron estudios dirigidos a personas con ocupaciones diversas entre las que destacan: empleados de atención al cliente, profesores, oficiales, trabajadores de servicio de alimentos y cuidadores. El origen de los participantes varió en términos de la nacionalidad y la etnicidad, registrándose entre los grupos estudiados bajo el primer rubro a subgrupos de italianos, nacionales de otros países europeos no especificados, japoneses, coreanos y bajo el segundo rubro de etnicidad a afroamericanos, coreanoamericanos e hispanos radicados en EE. UU.
Es importante mencionar que aunque las intervenciones del estudio están enfocadas principalmente a la reducción y manejo de estrés con principal relación a la hipertensión, muchos de los participantes aparte de padecer de la presión arterial reportaron tener otro tipo de enfermedades (22\% de los mismos tienen relación con enfermedades coronarias, cáncer, VIH, estrés postraumático, síndrome del intestino irritable y enfermedades relacionadas con el estrés), sin embargo, esto no interfirió, pues prácticamente se buscaba el control de estrés para aminorar la presencia de la presión arterial.

El tipo de intervenciones que se identificaron para el manejo y control de estrés fueron: cognitiva con un $10 \%$ de aplicación en los estudios examinados, cognitiva- conductual (26\%), conductual (38\%), preventiva primaria y secundaria (2\%), preventiva primaria( $4 \%$ ), promocional (2\%), multifactorial (4\%), multinivel basada en la evidencia (2\%), No farmacológica y farmacológica (2\%) y secuencial y simultánea (2\%). De esta distribución porcentual, es evidente la preponderancia de los estudios conductuales, aunque vale la pena subrayar que las categorías del tipo de intervención se recogieron de los propios artículos y aunque no fueron excluyentes entre sí en algunos casos, como por ejemplo los estudios multifactoriales, los preventivos, los promocionales, los multinivel y los no farmacológicos, esta clasificación brinda una idea general del énfasis central registrado en los estudios de intervención psicológica contra la hipertensión. 
En la tabla 2 se presenta con más detalle el total de 50 artículos objeto del presente análisis crítico, organizados a partir del tipo de intervención, las técnicas involucradas, el número de sesiones y los resultados principales del procedimiento, identificando a los estudios por su primer autor y el año de publicación del artículo.

La tabla 2 presenta datos específicos de cada artículo que permiten hacer un análisis comparativo de los mismos en términos de diferentes criterios, como duración de las intervenciones y éxito relativo de las mismas. El número de sesiones de los estudios analizados varió de 1 a 20, con un tiempo que osciló de 15' a 90' por sesión, y una distribución de las sesiones en un lapso que varió entre de día a 12 meses. Las actividades específicas empleadas en las sesiones fueron desde una exposición a estímulos visuales estresantes por 1', hasta actividades diversas desarrolladas en un tiempo indeterminado no mayor a 90'.

En cuanto a los resultados obtenidos con las intervenciones aplicadas en cada estudio se encontraron los siguientes: 1) Con la intervención cognitiva, misma que se empleó en 5 estudios; se logró la disminución de la depresión y el estrés y bienestar emocional con el uso continuo de las competencias adquiridas y el afrontamiento, tanto a nivel individual como grupal. 2) Con la intervención cognitivo-conductual la cual se empleó en 13 estudios; hubo una reducción significativa de la presión arterial, a través del entrenamiento del manejo de estrés, también tuvo un impacto positivo en la disminución de las quejas psicoló- gicas, disminución en los niveles de estrés, aumento de la atención plena, reducción de ansiedad y de depresión, y una mejora de la capacidad percibida. 3) Con la intervención conductual empleada en 19 estudios, hubo un incremento en los niveles de confianza, mejorando sus hábitos de comunicación y logrando reducir los trastornos psicológicos, así como la frecuencia cardiaca y presión arterial; así como también se lograron efectos favorables en el conocimiento y habilidades de afrontamiento, disminución en la depresión y reducción en los niveles de estrés y de tensión. 4) Con la intervención preventiva, empleada en siete estudios, se brindó información médica precisa, sobre el manejo de la hipertensión y la modificación de estilos de vida logrando un efecto preventivo beneficioso para la salud física de los participantes. 5) Con la intervención promocional, empleada únicamente en un estudio, se redujo la angustia psicológica y aumentó la motivación de los participantes. 6) Con la intervención multifactorial, la cual fue empleada en un estudio, hubo una mejora en los hábitos dietéticos, un aumento en la frecuencia del ejercicio, propiciando un mejor control de la salud en general, mostrando una reducción en los síntomas del estrés. 7) La intervención multinivel empleada en un estudio, resultó eficaz para el control de la presión arterial. 8) Por último, la intervención secuencial y simultánea utilizada en un estudio, fue poco eficaz, pues permitió la interferencia de diversos obstáculos, como la adicción al cigarro (Véase la tabla 2).

Tabla 2. Técnicas, duración de las sesiones y resultados de investigaciones para el manejo y reducción de estrés asociado a hipertensión en investigaciones realizadas en el periodo 2000-2013.

\begin{tabular}{|c|c|c|c|c|}
\hline $\begin{array}{l}\text { Tipo de } \\
\text { intervención }\end{array}$ & Técnicas empleadas & $\begin{array}{l}\text { Primer } \\
\text { autor y año }\end{array}$ & $\begin{array}{l}\text { N. }{ }^{\circ} \text { de } \\
\text { sesiones }\end{array}$ & Resultados \\
\hline Cognitiva & $\begin{array}{l}\text { Programa de } \\
\text { reducción de estrés } \\
\text { basado en atención } \\
\text { plena }\end{array}$ & $\begin{array}{l}\text { Lubow, G. } \\
2011\end{array}$ & $\begin{array}{l}\text { Ocho sesiones } \\
\text { semanales de } \\
90\end{array}$ & $\begin{array}{l}\text { La depresión y el estrés percibido } \\
\text { disminuyeron durante la intervención, los } \\
\text { participantes informaron el continuo uso de } \\
\text { las competencias adquiridas. }\end{array}$ \\
\hline Cognitiva & $\begin{array}{l}\text { Terapia de } \\
\text { procesamiento } \\
\text { cognitivo. }\end{array}$ & $\begin{array}{l}\text { Walter, K., } \\
2012\end{array}$ & $\begin{array}{l}12 \text { sesiones de } \\
\text { grupo de } 90 ’\end{array}$ & $\begin{array}{l}\text { Los veteranos pueden beneficiarse con la } \\
\text { terapia de procesamiento cognitivo tanto a } \\
\text { nivel individual como grupal. }\end{array}$ \\
\hline \multirow[t]{2}{*}{ Cognitiva } & $\begin{array}{l}\text { Manejo de } \\
\text { estrés basado en } \\
\text { autosugestión. }\end{array}$ & $\begin{array}{l}\text { Munz, D. } \\
2001\end{array}$ & Tres meses. & $\begin{array}{l}\text { Las personas que asistieron a la formación } \\
\text { de autogestión mejoraron en bienestar } \\
\text { emocional, la productividad se incrementó y } \\
\text { el ausentismo disminuyó durante el período. }\end{array}$ \\
\hline & & & & Esta tabla continúa en la siguiente página $\longrightarrow$ \\
\hline
\end{tabular}




\begin{tabular}{|c|c|c|c|c|}
\hline $\begin{array}{l}\text { Tipo de } \\
\text { intervención }\end{array}$ & Técnicas empleadas & $\begin{array}{l}\text { Primer } \\
\text { autor y año }\end{array}$ & $\begin{array}{l}\mathrm{N} .{ }^{\circ} \text { de } \\
\text { sesiones }\end{array}$ & Resultados \\
\hline Cognitiva & Reducción del estrés & $\begin{array}{l}\text { Townsend, } \\
\text { T. } 2007\end{array}$ & Ocho semanas & $\begin{array}{l}\text { No se observaron reducciones en } \\
\text { depresión, estrés y afrontamiento del pre al } \\
\text { postest, aunque el afrontamiento fue más } \\
\text { significativo. }\end{array}$ \\
\hline Cognitiva & $\begin{array}{l}\text { Reducción de estrés } \\
\text { consciente }\end{array}$ & $\begin{array}{l}\text { Bögels, S. } \\
2013\end{array}$ & No especifica & $\begin{array}{l}\text { Se redujo la internalización de los niños y } \\
\text { de los padres, tamaño del efecto medio y los } \\
\text { problemas exteriorizados de los niños, con } \\
\text { mejoras significativas en estrés de padres e } \\
\text { integridad de la familia }\end{array}$ \\
\hline $\begin{array}{l}\text { Cognitivo- } \\
\text { conductual }\end{array}$ & $\begin{array}{l}\text { Reducción de estrés } \\
\text { Tinnitus }\end{array}$ & $\begin{array}{l}\text { Gans, J. } \\
2013\end{array}$ & Ocho sesiones & $\begin{array}{l}\text { Estudios clínicamente significativos y con } \\
\text { aumento sustancial del funcionamiento } \\
\text { social, así un aumento global moderado en } \\
\text { Salud Mental. }\end{array}$ \\
\hline $\begin{array}{l}\text { Cognitivo- } \\
\text { conductual }\end{array}$ & $\begin{array}{l}\text { Entrenamiento } \\
\text { respiratorio breve en } \\
\text { Baroreceptor Cardiac } \\
\text { Reflex. }\end{array}$ & $\begin{array}{l}\text { Reyes, G. } \\
2006\end{array}$ & Una sesión. & $\begin{array}{l}\text { Se redujeron la frecuencia respiratoria, } \\
\text { periodo inspiratorio y el periodo } \\
\text { espiratorio durante el periodo de prácticas } \\
\text { respiratorias. }\end{array}$ \\
\hline $\begin{array}{l}\text { Cognitivo- } \\
\text { conductual }\end{array}$ & $\begin{array}{l}\text { Manejo de estrés, } \\
\text { cognitivo-conductual } \\
\text { y entrenamiento de } \\
\text { manejo de estrés }\end{array}$ & $\begin{array}{l}\text { Vocks, S. } \\
2004\end{array}$ & $\begin{array}{l}\text { Seis sesiones } \\
\text { de } 90 \text { '. }\end{array}$ & $\begin{array}{l}\text { Reducción significativa de presión arterial, } \\
\text { vía entrenamiento de manejo del estrés. } \\
\text { También la radioactividad del sistema } \\
\text { cardiovascular se mitigó vía intervenciones } \\
\text { cognitivo-conductuales. }\end{array}$ \\
\hline $\begin{array}{l}\text { Cognitivo- } \\
\text { conductual }\end{array}$ & $\begin{array}{l}\text { Manejo de estrés } \\
\text { cognitivo-conductual, } \\
\text { técnicas de } \\
\text { afrontamiento, terapia } \\
\text { racional emotiva, } \\
\text { razonamiento } \\
\text { irracional; ejercicio y } \\
\text { relajación. }\end{array}$ & $\begin{array}{l}\text { Rhenen, W. } \\
2005\end{array}$ & No especifica & $\begin{array}{l}\text { Ambas intervenciones revelaron un impacto } \\
\text { positivo en las quejas psicológicas. }\end{array}$ \\
\hline $\begin{array}{l}\text { Cognitivo- } \\
\text { conductual }\end{array}$ & $\begin{array}{l}\text { Manejo de estrés } \\
\text { con base cognitivo- } \\
\text { conductual }\end{array}$ & $\begin{array}{l}\text { Penedo, F. } \\
2006\end{array}$ & 10 semanas. & $\begin{array}{l}\text { Aumento de la calidad de vida con el } \\
\text { desarrollo de gestión de habilidades del } \\
\text { estrés. }\end{array}$ \\
\hline $\begin{array}{l}\text { Cognitivo- } \\
\text { conductual }\end{array}$ & $\begin{array}{l}\text { Programa de } \\
\text { reducción de estrés } \\
\text { basado en atención } \\
\text { plena }\end{array}$ & $\begin{array}{l}\text { Carmody, J. } \\
2008\end{array}$ & Ocho semanas. & $\begin{array}{l}\text { Los niveles de estrés percibidos } \\
\text { disminuyeron y los de atención aumentaron. } \\
\text { La práctica de ejercicios de meditación se } \\
\text { relacionó con el cambio en la mayoría de } \\
\text { facetas de atención. }\end{array}$ \\
\hline $\begin{array}{l}\text { Cognitivo- } \\
\text { conductual }\end{array}$ & $\begin{array}{l}\text { Programa de } \\
\text { reducción de estrés } \\
\text { basado en atención } \\
\text { plena }\end{array}$ & $\begin{array}{l}\text { Gold, E. } \\
2010\end{array}$ & $\begin{array}{l}\text { Ocho a nueve } \\
\text { sesiones } \\
\text { semanales de } \\
2.5 \text { hrs. }\end{array}$ & $\begin{array}{l}\text { Los participantes sufrían de angustia } \\
\text { emocional, asociada con deficiencias } \\
\text { de motivación, concentración y falta de } \\
\text { confianza en sí mismos. Posteriormente } \\
\text { experimentaron reducción de estrés, } \\
\text { ansiedad y depresión. }\end{array}$ \\
\hline
\end{tabular}




\begin{tabular}{llll}
\hline \hline $\begin{array}{l}\text { Tipo de } \\
\text { intervención }\end{array}$ & Técnicas empleadas & $\begin{array}{l}\text { Primer } \\
\text { autor y año }\end{array}$ & $\begin{array}{l}\text { N. }{ }^{\circ} \text { de } \\
\text { sesiones }\end{array}$ \\
\hline $\begin{array}{l}\text { Cognitivo- } \\
\text { conductual }\end{array}$ & $\begin{array}{l}\text { Programa de } \\
\text { reducción de estrés } \\
\text { basado en atención } \\
\text { plena }\end{array}$ & $\begin{array}{l}\text { Campbell, } \\
\text { T. 2011 }\end{array}$ & $\begin{array}{l}\text { Ocho sesiones } \\
\text { semanales de } \\
90 ' .\end{array}$ \\
$\begin{array}{l}\text { Cognitivo- } \\
\text { conductual }\end{array}$ & $\begin{array}{l}\text { Intervención grupal } \\
\text { cognitivo conductual }\end{array}$ & Phillips, K. & $\begin{array}{l}\text { 6 a } 12 \text { meses } \\
\text { de duración. }\end{array}$
\end{tabular}

Resultados

El programa MBSR fue eficaz en la reducción de la rumia y mejoró la atención consciente en pacientes con cáncer, sin embargo, su utilidad para reducir los niveles de presión arterial requiere una evaluación más a fondo.

Mejoró la capacidad percibida para relajarse y utilizar habilidades cognitivas de reevaluación a los seis meses de la intervención y una reducción de cortisol de 6 a 12 meses.

\section{Cognitivo- conductual \\ Intervención grupal basada en el Programa de reducción de estrés basado en atención plena \\ Cognitivo- conductual \\ Cognitivo- conductual \\ Cognitivo- conductual \\ Gayner, B. 2012 \\ Morone, N. Ocho semanas 2012 \\ Reginald, D. 2012 \\ Nueve semanas. \\ Cruess, S. 10 semanas 2002 cognitivo-conductual sobre el estado de ánimo, síntomas depresivos}

Conductual Reducción de estrés

\section{H.} 2000

Conductual Reducción de estrés a Barnes, V. través de la relajación 2003 mental

Cognitivoconductual

Winefield, Ocho semanas

Programa de reducción de estrés basado en atención plena.

Sesiones diarias de 15, en casa y en la escuela, durante cuatro meses. 2012
El aumento de la atención plena se correlacionó con la reducción de la evasión, mayor afecto positivo y menos depresión, MBSR con efectos clínicamente significativos.

Los participantes percibieron una mejoría en su salud física y se comprobaron los beneficios del programa para la salud.

La intervención cognitivo-conductual redujo significativamente la gravedad del trastorno de estrés postraumático, depresión y ansiedad general.

El grupo (de manejo de estrés cognitivoconductual) demostró una reducción significativa de los trastornos psicológicos.

Los participantes incrementaron su confianza en sí mismos y mejoraron sus hábitos de comunicación.

El grupo presentó una media total en

Morone, N. Ocho semanas. Los participantes percibieron una mejoría reducción del 0.1 en infracciones, del 0.3 días de suspensión; del 6.4 en períodos cambios significativos en calificaciones. en su salud física y se comprobaron los beneficios del programa para la salud. de clase ausente y una 0.5 tardanzas; sin 


\begin{tabular}{|c|c|c|c|c|}
\hline $\begin{array}{l}\text { Tipo de } \\
\text { intervención }\end{array}$ & Técnicas empleadas & $\begin{array}{l}\text { Primer } \\
\text { autor y año }\end{array}$ & $\begin{array}{l}\text { N. }{ }^{\circ} \text { de } \\
\text { sesiones }\end{array}$ & Resultados \\
\hline $\begin{array}{l}\text { Cognitivo- } \\
\text { conductual }\end{array}$ & $\begin{array}{l}\text { Terapia cognitivo- } \\
\text { conductual y } \\
\text { terapia cognitiva sin } \\
\text { exposición }\end{array}$ & $\begin{array}{l}\text { Reginald, } \\
\text { D. } 2012\end{array}$ & $\begin{array}{l}\text { Nueve } \\
\text { semanas. }\end{array}$ & $\begin{array}{l}\text { La intervención cognitivo-conductual redujo } \\
\text { significativamente la gravedad del trastorno } \\
\text { de estrés postraumático, depresión y } \\
\text { ansiedad general. }\end{array}$ \\
\hline $\begin{array}{l}\text { Cognitivo- } \\
\text { conductual }\end{array}$ & $\begin{array}{l}\text { Manejo de estrés } \\
\text { cognitivo-conductual } \\
\text { sobre el estado de } \\
\text { ánimo, síntomas } \\
\text { depresivos }\end{array}$ & $\begin{array}{l}\text { Cruess, S. } \\
2002\end{array}$ & 10 semanas & $\begin{array}{l}\text { El grupo (de manejo de estrés cognitivo- } \\
\text { conductual) demostró una reducción } \\
\text { significativa de los trastornos psicológicos. }\end{array}$ \\
\hline Conductual & Reducción de estrés & $\begin{array}{l}\text { Winefield, } \\
\text { H. } \\
2000\end{array}$ & Ocho semanas & $\begin{array}{l}\text { Los participantes incrementaron su } \\
\text { confianza en sí mismos y mejoraron sus } \\
\text { hábitos de comunicación. }\end{array}$ \\
\hline Conductual & $\begin{array}{l}\text { Reducción de estrés a } \\
\text { través de la relajación } \\
\text { mental }\end{array}$ & $\begin{array}{l}\text { Barnes, V. } \\
2003\end{array}$ & $\begin{array}{l}\text { Sesiones diarias } \\
\text { de } 15 \text { ' en casa } \\
\text { y en la escuela, } \\
\text { durante cuatro } \\
\text { meses. }\end{array}$ & $\begin{array}{l}\text { El grupo presentó una media total en } \\
\text { reducción del } 0.1 \text { en infracciones, del } 0.3 \\
\text { días de suspensión; del } 6.4 \text { en períodos } \\
\text { de clase ausente y una } 0.5 \text { tardanzas; sin } \\
\text { cambios significativos en calificaciones. }\end{array}$ \\
\hline Conductual & $\begin{array}{l}\text { Retroalimentación } \\
\text { biológica }\end{array}$ & $\begin{array}{l}\text { Overhaus, } \\
\text { S. } 2003\end{array}$ & $\begin{array}{l}\text { Ocho sesiones } \\
\text { en dos } \\
\text { semanas }\end{array}$ & $\begin{array}{l}\text { Los participantes normotensos mostraron } \\
\text { valores significativamente más altos. No se } \\
\text { detectó ninguna reducción en la presión } \\
\text { arterial durante las sesiones y en promedio } \\
\text { la presión arterial se incrementó para ambos } \\
\text { grupos. }\end{array}$ \\
\hline Conductual & $\begin{array}{l}\text { Reducción de estrés. } \\
\text { Trato justo }\end{array}$ & $\begin{array}{l}\text { Vermunt, R. } \\
2003\end{array}$ & No especifica & $\begin{array}{l}\text { Las respuestas fisiológicas: frecuencia } \\
\text { cardíaca, sístole y presión arterial diástole, } \\
\text { disminuyeron significativamente después de } \\
\text { un trato justo en comparación con un trato } \\
\text { injusto. }\end{array}$ \\
\hline Conductual & $\begin{array}{l}\text { Técnica de } \\
\text { resolución de } \\
\text { problemas. }\end{array}$ & $\begin{array}{l}\text { Shimazu, A. } \\
2006\end{array}$ & $\begin{array}{l}\text { Una sesión } \\
\text { dividida en } \\
\text { cuatro partes } \\
\text { de } 20 \text { ', } 45 \text { ', } \\
\text { 45' y } 10 \text { '. }\end{array}$ & $\begin{array}{l}\text { Efectos favorables en conocimiento y } \\
\text { habilidades de afrontamiento, adquiridos } \\
\text { en una sesión de control de estrés, esta } \\
\text { intervención resultó más eficaz para } \\
\text { personas con un alto control de trabajo. }\end{array}$ \\
\hline Conductual & $\begin{array}{l}\text { Manejo de estrés } \\
\text { Afrontamiento y } \\
\text { comportamientos } \\
\text { saludables }\end{array}$ & $\begin{array}{l}\text { Carrico, A. } \\
2006\end{array}$ & 10 semanas. & $\begin{array}{l}\text { Disminución significativa en el ánimo } \\
\text { deprimido y la negación del afrontamiento } \\
\text { inmediatamente después de la intervención; } \\
\text { sin cambios en el comportamiento cognitivo } \\
\text { activo. }\end{array}$ \\
\hline Conductual & $\begin{array}{l}\text { Retroalimentación } \\
\text { biológica }\end{array}$ & $\begin{array}{l}\text { Overhaus, } \\
\text { S. } 2003\end{array}$ & $\begin{array}{l}\text { Ocho sesiones } \\
\text { en dos } \\
\text { semanas }\end{array}$ & $\begin{array}{l}\text { Los participantes normotensos mostraron } \\
\text { valores significativamente más altos. No se } \\
\text { detectó ninguna reducción en la presión } \\
\text { arterial durante las sesiones y en promedio } \\
\text { la presión arterial se incrementó para ambos } \\
\text { grupos. }\end{array}$ \\
\hline
\end{tabular}




\begin{tabular}{|c|c|c|c|c|}
\hline $\begin{array}{l}\text { Tipo de } \\
\text { intervención }\end{array}$ & Técnicas empleadas & $\begin{array}{l}\text { Primer } \\
\text { autor y año }\end{array}$ & $\begin{array}{l}\mathrm{N} .{ }^{\circ} \text { de } \\
\text { sesiones }\end{array}$ & Resultados \\
\hline Conductual & $\begin{array}{l}\text { Reducción de estrés. } \\
\text { Trato justo }\end{array}$ & $\begin{array}{l}\text { Vermunt, R. } \\
2003\end{array}$ & No especifica & $\begin{array}{l}\text { Las respuestas fisiológicas: frecuencia cardíaca, } \\
\text { sístole y presión arterial diástole, disminuyeron } \\
\text { significativamente después de un trato justo } \\
\text { en comparación con un trato injusto. }\end{array}$ \\
\hline Conductual & $\begin{array}{l}\text { Técnica de } \\
\text { resolución de } \\
\text { problemas. }\end{array}$ & $\begin{array}{l}\text { Shimazu, A. } \\
2006\end{array}$ & $\begin{array}{l}\text { Una sesión } \\
\text { dividida en } \\
\text { cuatro partes } \\
\text { de } 20 \text { ', } 45 \text { ', } \\
45^{\prime} \text { y } 10 \text { '. }\end{array}$ & $\begin{array}{l}\text { Efectos favorables en conocimiento y } \\
\text { habilidades de afrontamiento, adquiridos } \\
\text { en una sesión de control de estrés, esta } \\
\text { intervención resultó más eficaz para } \\
\text { personas con un alto control de trabajo. }\end{array}$ \\
\hline Conductual & $\begin{array}{l}\text { Manejo de estrés } \\
\text { Afrontamiento y } \\
\text { comportamientos } \\
\text { saludables }\end{array}$ & $\begin{array}{l}\text { Carrico, A. } \\
2006\end{array}$ & 10 semanas. & $\begin{array}{l}\text { Disminución significativa en el ánimo } \\
\text { deprimido y la negación del afrontamiento } \\
\text { inmediatamente después de la intervención; } \\
\text { sin cambios en el comportamiento cognitivo } \\
\text { activo. }\end{array}$ \\
\hline
\end{tabular}

Conductual Relajación y

disminución de ansiedad
Rausch, S. Tres formas 2008

\section{de relajación} de 20'. Cinco estímulos visuales estresantes de 1'. Relajación de 10'.

Conductual Motivación, técnica de grupos focales y juegos de rol.

Conductual Manejo de estrés (incluye relajación)

Conductual

Reducción de estrés. Atención de procesos de pensamiento de rumia

Conductual Retroalimentación biológica. Entrenamiento de relajación asistida

Conductual El Jhon Henryism
Blom, M., 2009

Scala, D., 2008

Sesiones de dos hrs., en 12 meses.

20 sesiones de dos hrs., en un año.

Labelle, L. 2010

Sesiones de 90' en ocho semanas.

Olsson, E. 2010

Clark, R. 2001
Los controles de manipulación de las intervenciones de relajación fueron eficaces, el estrés en general y los niveles de tensión disminuyeron sistemáticamente después de la relajación.

El enfoque motivacional disminuyó la presión arterial de manera significativa después de un año e influyó en los pacientes para modificar su estilo de vida.

El comportamiento de la tensión diaria se redujo con el tiempo, pronunciándose más en el grupo de intervención, sin embargo, no hubo diferencias significativas con el grupo control.

El efecto del tratamiento del grupo sobre el cambio en las puntuaciones de depresión fue significativo. La observación de pensamientos, emociones y sensaciones corporales, sin juicio, puede reducir la rumia y los síntomas depresivos.

El tratamiento permitió la reducción de la presión arterial sistólica y el promedio de cumplimiento de los pacientes en el tratamiento fue de 3.9.

Los tratamientos conductuales producen efectos moderadamente fiables para la reducción de la presión arterial. 


\begin{tabular}{|c|c|c|c|c|}
\hline $\begin{array}{l}\text { Tipo de } \\
\text { intervención }\end{array}$ & Técnicas empleadas & $\begin{array}{l}\text { Primer } \\
\text { autor y año }\end{array}$ & $\begin{array}{l}\mathrm{N} .{ }^{\circ} \text { de } \\
\text { sesiones }\end{array}$ & Resultados \\
\hline Conductual & $\begin{array}{l}\text { Técnicas cognitivas, } \\
\text { conductuales y } \\
\text { de comunicación } \\
\text { asertiva y positiva. }\end{array}$ & $\begin{array}{l}\text { Stauder, A., } \\
2010\end{array}$ & $12 \mathrm{hrs}$. & $\begin{array}{l}\text { El nivel del estrés y los síntomas somáticos } \\
\text { disminuyeron, el bienestar de los } \\
\text { participantes incrementó. Se confirman } \\
\text { efectos positivos a largo plazo de la } \\
\text { intervención conductual estandarizada. }\end{array}$ \\
\hline Conductual & $\begin{array}{l}\text { Reducción de estrés } \\
\text { y ansiedad. Qigong }\end{array}$ & $\begin{array}{l}\text { Chow, Y. } \\
2011\end{array}$ & 12 semanas & $\begin{array}{l}\text { En la semana ocho, los sujetos en general } \\
\text { disfrutaron de mejores estados de ánimo y } \\
\text { calidad de vida. En la semana } 12 \text { el grupo } \\
\text { de Qigong experimentó una disminución } \\
\text { significativa en los niveles de estrés y ansiedad. }\end{array}$ \\
\hline Conductual & Reducción de estrés & $\begin{array}{l}\text { Epstein- } \\
\text { Lubow, G., } \\
2011\end{array}$ & $\begin{array}{l}\text { Ocho semanas } \\
\text { con sesiones } \\
\text { de } 90^{\prime} .\end{array}$ & $\begin{array}{l}\text { La gravedad de la depresión disminuyó } \\
\text { durante la intervención, con un tamaño del } \\
\text { efecto estimado para la depresión durante } \\
\text { el tratamiento activo de } 0.29 \text {, lo que sugiere } \\
\text { leve mejora. }\end{array}$ \\
\hline Conductual & $\begin{array}{l}\text { Autoeficacia y } \\
\text { Autocuidado }\end{array}$ & $\begin{array}{l}\text { Warren- } \\
\text { Findlow, J. } \\
2011\end{array}$ & No especifica & $\begin{array}{l}\text { La adhesión al autocuidado para la } \\
\text { hipertensión varió, pues un 22\% denotó una } \\
\text { adherencia a una dieta baja en sal, un } 75 \% \\
\text { para no fumar y un } 58 \% \text { a la medicación. }\end{array}$ \\
\hline Conductual & $\begin{array}{l}\text { Retroalimentación } \\
\text { biológica }\end{array}$ & $\begin{array}{l}\text { Palomba, D. } \\
2011\end{array}$ & $\begin{array}{l}\text { Cuatro } \\
\text { sesiones }\end{array}$ & $\begin{array}{l}\text { La presión arterial y las reacciones del estrés } \\
\text { psicosocial se redujeron significativamente } \\
\text { en los participantes. }\end{array}$ \\
\hline Conductual & $\begin{array}{l}\text { Programa educativo } \\
\text { de salud mental }\end{array}$ & $\begin{array}{l}\text { Orth- } \\
\text { Gomér, K. } \\
2012\end{array}$ & $\begin{array}{l}10 \text { sesiones de } \\
\text { dos hrs. }\end{array}$ & $\begin{array}{l}\text { Las tensiones diarias de la vida y la ansiedad, } \\
\text { disminuyeron significativamente para } \\
\text { hombres y mujeres, ambos aspectos mayor } \\
\text { efecto en las mujeres. }\end{array}$ \\
\hline Preventiva & $\begin{array}{l}\text { Control de la } \\
\text { hipertensión }\end{array}$ & $\begin{array}{l}\text { Han, H.R. } \\
2007 .\end{array}$ & No especifica & $\begin{array}{l}\text { La falta de medicación puede reducir el } \\
\text { control de la hipertensión. Es importante } \\
\text { proporcionar información médica precisa } \\
\text { sobre el manejo de la hipertensión y la } \\
\text { modificación de estilos de vida, incluyendo } \\
\text { ejercicio moderado, reducción de cigarrillos } \\
\text { e ingesta de alcohol. }\end{array}$ \\
\hline Preventiva & $\begin{array}{l}\text { Estrategias de } \\
\text { modificación de dieta } \\
\text { y reducción de estrés. }\end{array}$ & $\begin{array}{l}\text { Aroian, K. } \\
2012\end{array}$ & $\begin{array}{l}\text { Una sesión de } \\
\text { grupo de 90'. }\end{array}$ & $\begin{array}{l}\text { Actitud positiva hacia la prevención de } \\
\text { la hipertensión, sin embargo, no tienen } \\
\text { tiempo para preparar comidas saludables ni } \\
\text { para hacer ejercicio. }\end{array}$ \\
\hline Preventiva & $\begin{array}{l}\text { Programa de } \\
\text { reducción de estrés } \\
\text { basado en atención } \\
\text { plena AP, Terapia } \\
\text { Cognitiva Basada en } \\
\text { AP y Formación para } \\
\text { adultos }\end{array}$ & $\begin{array}{l}\text { Van der } \\
\text { Weijer- } \\
\text { Bergsma, E. } \\
2012\end{array}$ & $\begin{array}{l}12 \text { sesiones } \\
\text { de } 30 \text { ', en seis } \\
\text { semanas. }\end{array}$ & $\begin{array}{l}\text { Efecto preventivo sobre el estrés, el } \\
\text { bienestar y comportamiento de los } \\
\text { escolares. }\end{array}$ \\
\hline
\end{tabular}




\begin{tabular}{|c|c|c|c|c|}
\hline $\begin{array}{l}\text { Tipo de } \\
\text { intervención }\end{array}$ & Técnicas empleadas & $\begin{array}{l}\text { Primer } \\
\text { autor y año }\end{array}$ & $\begin{array}{l}\mathrm{N} .{ }^{\circ} \text { de } \\
\text { sesiones }\end{array}$ & Resultados \\
\hline Preventiva & $\begin{array}{l}\text { Reducción de estrés, } \\
\text { afrontamiento y } \\
\text { bienestar físico }\end{array}$ & $\begin{array}{l}\text { Anshel, M. } \\
2013\end{array}$ & $\begin{array}{l}10 \text { sesiones } \\
\text { semanales. }\end{array}$ & $\begin{array}{l}\text { Mejoría significativa tanto en la parte } \\
\text { inferior como en la parte superior del } \\
\text { cuerpo. }\end{array}$ \\
\hline $\begin{array}{l}\text { Prevención } \\
\text { primaria }\end{array}$ & $\begin{array}{l}\text { Entrenamiento } \\
\text { aeróbico }\end{array}$ & $\begin{array}{l}\text { Ben, D. } \\
2007\end{array}$ & $\begin{array}{l}\text { Sesiones } \\
\text { diarias de 30'. }\end{array}$ & $\begin{array}{l}\text { El ejercicio regular contribuye a } \\
\text { un envejecimiento saludable, el } \\
\text { entrenamiento aeróbico es muy eficaz en } \\
\text { la reducción de presión arterial en sujetos } \\
\text { normotensos. }\end{array}$ \\
\hline $\begin{array}{l}\text { Prevención } \\
\text { primaria }\end{array}$ & $\begin{array}{l}\text { Programa Educoeur } \\
\text { interdisciplinario }\end{array}$ & $\begin{array}{l}\text { Goyer, L. } \\
2013\end{array}$ & $\begin{array}{l}12 \text { y } 24 \\
\text { semanas }\end{array}$ & $\begin{array}{l}\text { Reducción significativa de riesgo } \\
\text { cardiovascular, peso, índice de masa } \\
\text { corporal, circunferencia de la cintura, } \\
\text { presión arterial sistólica, sus niveles de } \\
\text { estrés y mejoraron la salud mental. }\end{array}$ \\
\hline $\begin{array}{l}\text { Prevención } \\
\text { primaria y } \\
\text { secundaria }\end{array}$ & $\begin{array}{l}\text { Programa de cambio } \\
\text { estilo de vida }\end{array}$ & $\begin{array}{l}\text { Dau- } \\
\text { benmier, J. } \\
2007\end{array}$ & Tres meses. & $\begin{array}{l}\text { Mejoría global del riesgo coronario. } \\
\text { Reduciendo en la dieta la ingesta de } \\
\text { grasas, predijo reducciones en el peso, } \\
\text { el total de colesterol, interactuado con } \\
\text { el aumento de ejercicio para predecir la } \\
\text { reducción del estrés. }\end{array}$ \\
\hline Promocional & $\begin{array}{l}\text { Programa «Poder } \\
\text { para el cambio } \\
\text { Performance» }\end{array}$ & $\begin{array}{l}\text { McCraty, R. } \\
2009\end{array}$ & Tres meses. & $\begin{array}{l}\text { Mejoras significativas en colesterol, } \\
\text { glucosa, frecuencia cardíaca, presión } \\
\text { arterial, una actitud positiva, se redujo la } \\
\text { angustia psicológica general y aumento } \\
\text { significativamente la productividad, la } \\
\text { motivación, la claridad meta y el apoyo } \\
\text { percibido. }\end{array}$ \\
\hline $\begin{array}{l}\text { Multifac- } \\
\text { torial }\end{array}$ & $\begin{array}{l}\text { Programa estilo de } \\
\text { vida multifactorial }\end{array}$ & $\begin{array}{l}\text { Sudin, Ö. } \\
2003\end{array}$ & Siete días. & $\begin{array}{l}\text { Mejora saludable en los hábitos dietéticos, } \\
\text { frecuencia de ejercicio y un mejor control } \\
\text { de la salud en general. }\end{array}$ \\
\hline $\begin{array}{l}\text { Multifac } \\
\text { torial }\end{array}$ & $\begin{array}{l}\text { Programa de } \\
\text { reducción de estrés } \\
\text { basado en atención } \\
\text { plena (MBSR) }\end{array}$ & $\begin{array}{l}\text { Zernicke, } \\
\text { K. } 2012 \text {. }\end{array}$ & $\begin{array}{l}\text { Ocho sesiones } \\
\text { semanales de } \\
90\end{array}$ & $\begin{array}{l}\text { Se redujo la gravedad de los síntomas } \\
\text { del síndrome del intestino irritable y } \\
\text { síntomas de estrés, manteniéndose a los } \\
\text { seis meses posteriores de la intervención. } \\
\text { La atención, la automonitorización y la } \\
\text { anticipación de MBSR, pueden generar } \\
\text { pequeñas mejoras. }\end{array}$ \\
\hline $\begin{array}{l}\text { Multinivel } \\
\text { basada en la } \\
\text { evidencia }\end{array}$ & $\begin{array}{l}\text { Educación interactiva } \\
\text { para la hipertensión } \\
\text { y asesoramiento } \\
\text { conductual. }\end{array}$ & $\begin{array}{l}\text { Fernández, } \\
\text { S. } 2011\end{array}$ & 12 meses. & $\begin{array}{l}\text { Las intervenciones basadas en la evidencia } \\
\text { resultan efectivas para el control de la } \\
\text { presión arterial cuando se implementan } \\
\text { en centros médicos que atienden un alto } \\
\text { número de hipertensos de bajos ingresos y } \\
\text { con significativas barreras para el cambio de } \\
\text { comportamiento. }\end{array}$ \\
\hline
\end{tabular}

Esta tabla continúa en la siguiente página $\longrightarrow$ 


\begin{tabular}{lllll}
\hline \hline $\begin{array}{l}\text { Tipo de } \\
\text { intervención }\end{array}$ & Técnicas empleadas & $\begin{array}{l}\text { Primer } \\
\text { autor y año }\end{array}$ & $\begin{array}{l}\text { N. }{ }^{\circ} \text { de } \\
\text { sesiones }\end{array}$ & Resultados \\
\hline $\begin{array}{l}\text { No farmaco- } \\
\text { lógica y } \\
\text { farmaco- } \\
\text { lógica }\end{array}$ & Actividad física & $\begin{array}{l}\text { Beckett, N. } \\
2009\end{array}$ & $\begin{array}{l}\text { Seis sesiones } \\
\text { mensuales de } \\
\text { grupo. }\end{array}$ & $\begin{array}{l}\text { El tratamiento de la hipertensión en la } \\
\text { edad avanzada resulta ser benéfico para la } \\
\text { reducción de la morbilidad y mortalidad } \\
\text { cardiovascular, además de que reduce el } \\
\text { riesgo de demencia. }\end{array}$ \\
$\begin{array}{l}\text { Secuencial y } \\
\text { simultanea }\end{array}$ & $\begin{array}{l}\text { Psicoterapia, } \\
\text { asesoramiento y } \\
\text { paquetes educativos }\end{array}$ & $\begin{array}{l}\text { Mansyur, C. } \\
2013\end{array}$ & $\begin{array}{l}\text { Tres visitas } \\
\text { programadas } \\
\text { cada seis } \\
\text { meses. }\end{array}$ & $\begin{array}{l}\text { Varios obstáculos interfirieron para } \\
\text { modificar el comportamiento, entre ellas la } \\
\text { adicción al cigarro. }\end{array}$ \\
& & & \\
\hline \hline
\end{tabular}

Finalmente, es importante mencionar que en las intervenciones se encontraron diferentes obstáculos que interfirieron en la modificación de la conducta, como: la adicción al cigarro, la falta de tiempo para preparar alimentos saludables y para hacer ejercicio. Por otro lado, las intervenciones tuvieron resultados favorables en la mayoría de las poblaciones, aunque se hace referencia que sus efectos beneficiaron aún más a los veteranos en comparación con otras poblaciones. De igual manera, los estudios mostraron evidencia de que hay más inclinación hacia las intervenciones de tipo conductual, aunque las que más contribuyen a la disminución de los niveles de estrés y por ende a una reducción significativa de la presión arterial fueron las intervenciones cognitivo-conductuales, esto tomando en cuenta que el 100\% (13) de los estudios basados en ese tipo de intervención, arrojaron resultados positivos, mientras que en los de tipo exclusivamente conductual (19) se reportó un $11.11 \%$ de resultados desfavorables y solo un $68.4 \%$ produjo una mejoría en la población objeto. A pesar de que los estudios no emplearon medidas equiparables para valorar el grado de éxito de los procedimientos psicológicos para reducir los niveles percibidos de estrés, ni tampoco para medir el efecto que tuvo esto sobre la disminución de la hipertensión arterial, se puede resumir de manera preliminar, que los autores mismos reportaron diferentes niveles de éxito de sus intervenciones y este es un primer paso para dar cuenta de los resultados diferenciales de los procedimientos empleados. Este hallazgo preliminar sobre el mayor beneficio de las intervenciones cognitivo conductuales sobre las exclusivamente conductuales puede deberse a que la primera es más integral que la segunda, al enfocarse no solamente a la disminución de comportamientos de riesgo para la salud, sino también a aspectos emocionales y de comportamiento verbal privado que son eficaces para mantener los estilos de vida saludables y disminuir la probabilidad de conductas de riesgo.

\section{Discusión}

El presente estudio se dio a la tarea de hacer una revisión de un grupo de artículos basados en intervenciones para el control y manejo del estrés en pacientes con hipertensión publicados en revistas científicas indizadas en la base bibliométrica Springer-Verlag, durante el periodo 2000-2013.

Hay que resaltar, que no sólo la hipertensión arterial fue el tema de estudio, algunos investigadores han escudriñado en el mundo del estrés, en relación con la hipertensión arterial y en general su relación con el riesgo de muerte cardiovascular.

Es relevante mencionar, «que gran variedad de investigadores han enfatizado la relación entre el estrés laboral y el aumento del riesgo de enfermedades cardiovasculares, las cuales demostraron que los efectos perjudiciales del estrés, unido a un día estresante de trabajo, parcialmente mediados por un aumento en la reactividad cardíaca, producen un aumento en la frecuencia cardíaca, la tensión arterial y una disminución del tono vagal, llevando finalmente al desarrollo lento y progresivo de la hipertensión arterial» referido por Virijkotte (2000; citado en Álvarez et ál., 2004, p. 18).

Tal situación, tiene semejanza con lo que se encontró en algunas de las investigaciones analizadas, las 
cuales en su mayoría se centraron en personas con actividad laboral, como cuidadores de pacientes psiquiátricos (Winefield, 2000) empleados de servicio al cliente de una empresa (Munz, Kohler \& Greenberg, 2001) empleados de una empresa japonesa (Shimazu, Umanodan \& Schaufeli, 2006), oficiales correccionales (McCarty, Atkinson \& Lipsenthal, 2009) profesores de primaria y auxiliares docentes (Gold, Smith, Hopper, Herne, Glenis \& Hulland, 2010) cuidadores de ancianos (Lubow, McBee, Darling, Armey \& Miller, 2011) y profesionistas y trabajadores de servicio de alimentos (Aroian, Peters, Rudner \& Waser, 2012).

Por otro lado, Gutiérrez (2001, p. 100) expone que «el estrés puede ser un factor de riesgo cardiovascular y su manejo podría ser beneficioso para el paciente hipertenso». Tal planteamiento nos da pie a considerar que el manejo del estrés puede tener resultados beneficiosos tanto para la salud en general, como para la reducción de la presión arterial.

A partir de la revisión realizada, se encontró que las intervenciones más eficaces para contrarrestar los efectos negativos que el estrés tiene en la salud de los hipertensos fueron las terapias cognitivo-conductuales, lo cual coincide con lo expuesto por Fernández (2009), quien indica que los programas de intervención cognitivo-conductual que se han aplicado al tratamiento de enfermedades de tipo coronario (entre ellas la hipertensión arterial), han mostrado una eficacia elevada.

Por otro lado Miguel-Tobal, Cano-Vindel, Casado y Escalona (1994), tras realizar un estudio en el que se implementó un programa cognitivo-conductual, donde combinaron distintas técnicas terapéuticas tales como sesiones informativas, entrenamiento en relajación muscular progresiva, técnicas de respiración, solución de problemas y técnicas de autocontrol para el cambio de hábitos de vida, obtuvieron como resultado cambios en un buen número de las variables psicológicas tratadas (ansiedad, ira, solución de problemas, etc.), así como fuertes reducciones en los niveles de presión arterial. Dicha afirmación ha sido apoyada en el presente estudio por Cruess et ál. (2002), Vocks, Ockenfels, Jürgensen, Mussgay y Rüddel (2004), Rhenen, Blonk, Dijk y Shaufeli (2005), Reyes et ál. (2006), Penedo et ál. (2006), Carmody y Baer (2008), Gold, Smith, Hopper, Herne, Glenis y Hulland (2010), Campbell, Labelle, Bacon y Carlson (2011), Phillips et ál. (2011), Gayner et ál. (2012), Morone, Lynch, Losasso, Liebe y Greco (2012), Reginald, Sterk y Pearce (2012) y Gans, O’Sullivan y Bircheff (2013).

Un aspecto identificado, en revisiones realizadas por Molerio, Arce, Otero y Nieves (2005), es que las estrategias de afrontamiento que empleaban los sujetos para enfrentar las situaciones estresantes en 1998, estaban más centradas en comportamientos de tipo cognitivo y conductual; actualmente se lleva a cabo la aplicación de otros tipos de intervención, los cuales utilizan una gran diversidad de técnicas adicionales a las que se identifican tradicionalmente con las cognitivo-conductuales, que si bien no han alcanzado los resultados esperados, evidencian el interés y trabajo que tanto la Medicina como la Psicología están realizando para disminuir o erradicar este tipo de problema de salud que afecta a diversos sectores de la población.

Tomando en cuenta lo anterior, como consecuencia se puede afirmar que todas las conclusiones apuntan a la necesidad de cambios en las conductas individuales y en los estilos de vida, construyendo ambientes más propicios para la salud, a fin de influir benéficamente en las variaciones en el estrés y en la tensión arterial, tal como lo mencionan Lindquist, Berlín y Knuiman (1996).

Los resultados de los estudios revisados en este artículo respaldan la eficacia de las intervenciones cognitivo-conductuales para el control y manejo del estrés, reduciendo con ello los niveles de presión arterial.

Si bien los resultados de la revisión muestran evidencias de la eficacia de las intervenciones cognitivoconductuales, es importante tomar en consideración que existen obstáculos que impiden a los enfermos controlar su enfermedad o acceder a un nivel de vida más saludable, entre estos se encuentra el desconocimiento general de herramientas conductuales y la falta de adherencia al tratamiento, cuyas principales causas, de acuerdo con lo publicado en el III Foro Diálogos Pfizer-Pacientes son: instrucciones insuficientes, falló en la relación médico-paciente, desacuerdo del paciente respecto al tratamiento, mala memoria o efectos adversos al medicamento (Pfizer, 2009).

De los resultados de la presente revisión, surge un punto relevante como objeto de reflexión sobre la eficacia diferencial de los estudios analizados. El tiempo de apli- 
cación fluctuó en un rango muy amplio, lo que hace sospechar que de ese factor pudo depender el éxito del procedimiento; ya que no todas las personas reaccionan de la misma manera ante los tratamientos y unas requieren de mayor tiempo de exposición a las técnicas de intervención para alcanzar un cambio clínicamente significativo. Aunado a esto, habría que considerar la efectividad de las intervenciones cortas, por ejemplo de uno o dos días, con respecto a las que se han aplicado durante todo un año, ya que no se podría hacer una comparación entre ellas debido a la gran diferencia en términos de la saturación que pueden tener sobre los pacientes, con esas diferencias en exposición a los tratamientos. Dicho aspecto sin duda abre camino para nuevas investigaciones.

Finalmente, es importante considerar que todo tratamiento debe ajustarse a las necesidades individuales de los pacientes, a fin de que exista un balance de riesgo-beneficio adecuado, facilitándoles la información necesaria que les permita conocer los efectos adversos y la relevancia de cumplir con apego el tratamiento para obtener resultados óptimos en el control y disminución de su enfermedad.

\section{Referencias}

American Psychological Association (2013). Salud Mental/ Corporal: estrés. Recuperado el 14 de mayo de 2013 de: www.apa.org/centrodeapoyo/estres.aspx

Anshel, M., Umscheid, D. \& Brinthaupt, T. (2013). Effect of a combined coping skills and wellness program on perceived stress and physical energy among police emergency dispatchers: an exploratory study. Journal of Police and Criminal Psychology, 28, 1-14, available via: http://dx.doi.org/10.1007/ s11896-012-9110-x

Álvarez, D., Benavides, J., Bueno, O., Cuadros, V., Echeverry, D., Gómez, L., Guzmán, A., Torres, M., Aristizábal, F., \& Castaño, J. (2004). Estrés e hipertensión. Archivos de Medicina, 8, 13-21. Recuperado el 7 de junio de 2013 de: www.umanizales. edu.co/publicaciones/campos/medicina/archivos_ medicina/html/ediciones.html

Arrivillaga, Q., Varela, A., Cáceres de Rodríguez, D., Correa, S. \& Holguín, P. (2007). Eficacia de un programa cognitivo-conductual para la disminu- ción de los niveles de presión arterial. Pensamiento Psicológico, 3(9), 33-49.

Aroian, K., Peters, M., Rudner, N. \& Waser, L. (2012). Hypertension prevention beliefs of hispanics. Journal of Transcultural Nursing, 23(2), 134-142.

Barnes, V., Bauza, L. \& Treiber, F. (2003). Impact of stress reduction on negative school behavior in adolescents. Health and Quality of Life Outcomes, 1(10). Recuperado de www.hqlo.com/content/ pdf/1477-7525-1-10.pdf

Barnes, V. \& Orme, D. (2008). El impacto de la reducción del estrés en la hipertensión esencial y las enfermedades cardiovasculares. International Journal of Sport Science, 12(4), 1-30.

Beckett, N., \& Noimark, D. (2009). Hypertension therapy in the oldest patients. Informes Actuales de Riesgo Cardiovascular, 3, 239-246.

Ben, D. \& Oliveira, J. (2007). Hypertension in aging: physical activity as primary prevention. European Review of Aging and Physical Activity, 4, 85-89.

Blom, M., Georgiades, A., Janszky, I., Alinaghizadeh, H., Lindvall, B. \& Ahnve, S. (2009). Daily stress and social support among women with CAD: Results from a 1-year randomized controlled stress management intervention study. Journal of Behavioral Medicine, 16, 227-235, available via: http://dx.doi. org/10.1007/s12529-009-9031-y

Brisbon, N. M., \& Lowery, G. A. (2009). Mindfulness and levels of stress: a comparison of beginner and advanced hatha yoga practitioners. Journal of Religion and Health, 50, 931-941.

Bögels, S., Hellemans, J., Deursen, S., Romer, M. \& Meulen, R. (2013). Mindful parenting in mental health care: effects on parental and child psychopathology, parental stress, parenting, coparenting, and marital functioning. Mindfulness, 1, 107-120.

Campbell, T., Labelle, L., Bacon, S. \& Carlson, L. (2011). Impact of mindfulness-based stress reduction (MBSR) on attention, rumination and resting blood pressure in women with cancer: A waitlistcontrolled study. Journal of Behaivoral Medicine, 35, 262-271.

Carmody, J. \& Baer, R. (2008). Relationships between mindfulness practice and levels of mindfulness, medical and psychological symptoms and well- 
being in a mindfulness-based stress reduction program. Journal of Behavioral Medicine, 31, 23-33.

Carrico, A., Antoni, M., Durán, R., Ironson, G., Penedo, F., Fletcher, M., Klimas, N. \& Schneiderman, N. (2006). Reductions in depressed mood and denial coping during cognitive behavioral stress management with HIV-positive gay men treated with HAART. Annals of Behavioral Medicine, 31(2), 155-164.

Chow, Y.W., Dorcas, A. \& Siu, M. H. (2011). The effects of qigong on reducing stress and anxiety and enhancing body-mind well-being. Mindfulness, 3, 51-59

Clark, R., Adams, J. \& Clark, V. (2001). Effects of John Henrvism and anger-coping on mean arterial pressure changes in african american women. International Journal of Behavioral Medicine, 8(4), 270281.

Cruess, S., Antoni, M., Hayes, A., Penedo, F., Ironson, G., Fletcher, M., Lutgendorf, S. \& Schneiderman, N. (2002). Changes in mood and depressive symptoms and related change processes during cognitive-behavioral stress management in HIV-Infected Men. Cognitive Therapy and Research, 26(3), 373-392.

Daubenmier, J., Weidner, G. \& Sumner, M. (2007). The contribution of changes in diet, exercise, and stress management to changes in coronary risk in women and men in the multisite cardiac lifestyle intervention program. Annals of Behavioral Medicine, 33(1), 57-68.

Epstein-Lubow, G., McBee, L., Darling, E., Armey, M. \& Miller, I. (2011). A pilot investigation of mindfulness-based stress reduction for caregivers of Frail Elderly. Mindfulness, 2, 95-102. Available via: http://dx.doi.org/10.1007/s12671-011-0047-4

Fernández, V. (2009). Eficacia de una intervención cognitivoconductual en el tratamiento de la hipertensión arterial. Tesis doctoral. Facultad de Psicología, Universidad Complutense de Madrid, Madrid, España.

Fernández, S., Tobin, J., Cassells, A., Diaz, M., Kalida, C. \& Ogedegbe, G. (2011). The counseling african americans to control hypertension (CAATCH) trial: baseline demographic, clinical, psychosocial, and behavioral characteristics. Implementation Science, 6, 1-13.
Gans, J., O 'Sullivan, P. \& Bircheff, V. (2013). Mindfulness bases tinnitus stress reduction pilot study. Mindfulness, 3(4). Online first article, not assigned to an issue. Available via: http://dx.doi.org/10.1007/ s12671-012-0184-4.

Gayner, B., Esplen, M., DeRoche, P., Wong, J., Bishop, S., Kavanagh, L. \& Butler, K. (2012). A randomized controlled trial of mindfulness-based stress reduction to manage affective symptoms and improve quality of life in gay men living with HIV. Journal of Behavioral Medicine, 35, 272-285, available via: http://dx.doi.org/ 10.1007/s10865-011-9350-8

Gold, E., Smith, A., Hopper, I., Herne, D., Glenis, T. \& Hulland, C. (2010). Mindfulness-based stress reduction (MBSR) for primary school teachers. Journal of Child and Family Studies, 19, 184-189.

González, G. (2006). Manejo del estrés. Recuperado el 14 de mayo de 2013 de: http://books.google.com.mx/ books?id $=$ IWxbtjdHwZwC\&pg $=\mathrm{PA} 8 \& \mathrm{dq}=$ estr $\mathrm{es}+$ definicion\&hl $=\mathrm{es} 419 \& \mathrm{sa}=\mathrm{X} \& \mathrm{ei}=\mathrm{lMaSUZb}$ CB5TU9ATW9oDAAw\&ved=0CDAQ6AEwAA\# $\mathrm{v}=$ onepage $\& \mathrm{q}=$ estres $\% 20$ definicion $\& \mathrm{f}=$ false

Goyer, L., Dufour, R., Janelle, C., Blais, Ch., L'Abbe, C., Raymond, E., de Champlain, J. \& Larochelle, P. (2012). Randomized controlled trial on the longterm eficacy of a multifaceted, interdisciplinary lifestyle intervention in reducing cardiovascular risk and improving lifestyle in patients at risk of cardiovascular disease. Journal of Behavioral Medicine, 36, 212-224. Recuperado el 8 de abril de 2013, available via: http://dx.doi.org/10.1007/ s10865-012-9407-3

Gutiérrez, J. (2001). Tratamiento de la hipertensión arterial. Cambio de estilo de vida. Colombia Médica, 32(1), 99-102.

Han, H.R., Kim, K.B., Kang, J., Jeong, S., Kim, E. Y., \& Kim, M. T. (2007). Knowledge, beliefs, and behaviors about hypertension control among middle-aged Korean Americans with hypertension. Journal of Community Health, 32(5), 324-342.

Hekcler, E., Lambert, J., Leventhal, E., Jahn, E. \& Contrada, R. (2008). Commonsense illness beliefs, adherence behaviors, and hypertension control among African Americans. Journal of Behavioral Medicine, 31, 391-400. 
Holguín, L., Correa, D., Arrivillaga, M., Cáceres, D. \& Varela, M. (2006). Adherencia al tratamiento de hipertensión arterial: efectividad de un programa de intervención biopsicosocial. Universitas Psychologica, 5(3), 535-547.

Labelle, L. E., Campbell, T. S. \& Carlson, L. E., (2010). Mindfulness-based stress reduction in oncology: evaluating mindfulness and rumination as mediators of change in depressive symptoms. Mindfulness, 1, 28-40.

Lindquist, T. Berlin, L. \& Knuiman. M. (1996). Influence of lifestyle, coping and job stress on blood pressure in men and woman. Department of Medicine and Public Health, 29(1), 1-7.

Lubow, G., McBee, L., Darling, E., Armey, M. \& Miller, I. (2011). A pilot investigation of mindfulness-based stress reduction for caregivers of frail elderly. Mindfulness, 2, 95-102.

Mansyur, C., Pavlik, V., Hyman, D., Taylor, W. \& Goodrick, G. (2013). Self-efficacy and barriers to multiple behavior change in low-income African Americans with hypertension. Journal of Behavioral Medicine, 36, 75-85.

McCraty, R., Atkinson, M. \& Lipsenthal, L. (2009). New hope for correctional oficers: an innovative program for reducing stress and health risks Lourdes Arguelles. Applied Psychophysiology Biofeedback, 34, 251-272.

Molerio, O., Arce, M., Otero, I. \& Nieves, Z. (2005). El estrés como factor de riesgo de la hipertensión arterial esencial. Revista Cubana de Higiene y Epidemiología, 43(1), enero-abril. Recuperado en http:// bvs.sld.cu/revistas/hie/vol43_1_05/hie07105. htm, accedido en 13 junio, 2013.

Morone, N., Lynch, C., Losasso, V., Liebe, K. \& Greco, C. (2012). Mindfulness to reduce psychosocial stress. Mindfulness, 3(1), 22-29.

Munz, D., Kohler, J. \& Greenberg, C. (2001). Effectiveness of a comprehensive worksite stress management program: combining organizational and individual interventions. International Journal of Stress Management, 8(1). Retrieved at: www.workhealth. org/Work\%20and\%20Health\%2 0Class\%20 2009/12_Munz.pdf
Olsson, E., Alaoui, S., Carlberg, B., Carlbring, P. \& Ghaderi, A. (2010). Internet-based biofeedbackassisted relaxation training in the treatment of hypertension: a pilot study. Applied Psychophysiol Biofeedback, 35, 163-170.

Orth-Gomér, K. (2012). Behavioral interventions for coronary heart disease patients. BioPsychoSocial Medicine, 6(5), 1-7. Retrieved at: www.bpsmedicine. com/content/pdf/1751-0759-6-5.pdf

Overhaus, S., Rüddel, Curio, I., Mussgay, L. \& Scholz, B. (2003). Biofeedback of baroreflex sensitivity in patients with mild essential hypertension. International Journal of Behavioral Medicine, 10(1), 66-78.

Palomba, D., Ghisi, M,. Scozzari, S., Sarlo, M., Bonso, E., Dorigatti, F. \& Palatini, P. (2011). Biofeedback-assisted cardiovascular control in hypertensives exposed to emotional stress: a pilot. Applied Psychophysiology Biofeedback, 36, 185-192.

Penedo, F., Molton, I., Dahn, J., Shen, B., Kinsinger, D., Traeger, L., Siegel, S., Schneiderman, N. \& Antoni, M. (2006). A randomized clinical trial of group-based cognitive-behavioral stress management in localized prostate cancer: development of stress management skills improves quality of life and benefit finding. Annals of Behavioral Medicine, 31(3), 261-270.

Pfizer, (2009). III Foro Diálogos Pfizer-pacientes. Recuperado el 30 de abril del 2013 de: www.youtube.com/ watch ${ }_{\mathrm{v}}={ }_{\mathrm{vvAYDlccVf}} \mathrm{w}$

Phillips, K., Antoni, M., Carver, Ch., Lechner, S., Penedo, F., McCullough, M., Gluck, S., Derhagopian, R. \& Blomberg, B. (2011). Stress management skills and reductions in serum cortisol across the year after surgery for non-metastatic breast cancer. Cognitive Therapy and Research, 35, 595-600.

Rausch, S. M., Auerbarch, S. M. \& Gramling, S. E. (2008). Gender and ethic differences in stress reduction, reactivity, and recovery. Journal Sex Roles, 59, 726-737.

Reginald. D., Sterk, J. \& Pearce, A. (2012). Un ensayo aleatorio de la terapia cognitivo-conductual y la terapia cognitiva para los niños con trastorno de estrés postraumático después de un traumatismo único incidente. Journal of Abnormal Child Psychology, 40(3), 327-337. 
Reyes, G., Cea, J, González, A., Cabo, O., Caso, R., Martínez, B. \& Hernández, J. (2006). Short-term effects of a brief respiratory training on baroreceptor cardiac reflex function in normotensive and mild hypertensive subjects. Applied Psychophysiology and Biofeedback, 31(1), 37-49. Available via: http://dx.doi.org/10.1007/s10484-006-9003-9

Rhenen, W., Blonk, R., Dijk, F. \& Shaufeli, W. (2005). The effect of a cognitive and a physical stress-reducing programme on psychological complaints. International Archives of Occupational and Environmental Health, 78, 139-148.

Scala, D., D’Avino M., Cozzolino, S., Mancini, A., Andria, B., Caruso, G., Tajana, G. \& Caruso, D. (2008). Promotion of behavioural change in people with hypertension: an intervention study. Pharmacy World \& Science, 30, 834-839.

Shimazu, A., Umanodan, R. \& Schaufeli, W.B. (2006). Effects of a brief worksite stress management program on coping skills, psychological distress and physical complaints: a controlled trial. International Archives of Occupational and Environmental Health, 80, 60-69.

Stauder, A., Thege, B., Kovács, M., Balog, P., Williams, V. \& Williams, R. (2010). Worldwide stress: different problems, similar solutions? cultural adaptation and evaluation of a standardized stress management program in Hungary. International Journal of Behavioral Medicine, 17, 25-32.

Sundin, Ö., Lisspers, J., Hofman-Bang, C., Nygren, Å., Rydén, L. \& Öhman, A. (2003). Comparing multifactorial lifestyle interventions and stress management in coronary risk reduction. International Journal of Behavioral Medicine, 10(3), 191-204.

Miguel-Tobal, J.J., Cano-Vindel, A., Casado, M.I. y Escalona, A. (1994). Emociones e hipertensión. Implantación de un programa cognitivo-conductual en pacientes hipertensos. Anales de Psicología, 10(2), 199-216.
Townsend, T., Hawkins, S. \& Lanier, A. (2007). Stress and stress reduction among african american women: a brief report. Journal Primary Prevent, 28, 569-582.

Vermunt, R. \& Steensma, H. (2003). Physiological relaxation: stress reduction through fair treatment. Social Justice Research, 16(2), 135-149.

Vocks, S., Ockenfels, M., Jürgensen, R., Mussgay, L. \& Rüddel, H. (2004). Blood pressure reactivity can be reduced by a cognitive behavioral stress management program. International Journal of Behavioral Medicine, 11(2), 63-70.

Walter, K., Bolte, T., Owens, G. \& Chard, K. (2012). The impact of personality disorders on treatment outcome for veterans in a posttraumatic stress disorder residential treatment program. Cognitive Therapy and Research, 36, 576-584.

Warren-Findlow, J., Seymour, R. B. \& Brunner, L. R. (2011). The association between self-efficacy and hypertension self-care activities among african american adults. Journal of Community Health, 37, 15-24.

Van de Weijer-Bergsma, E., Langenberg, G., Brandsma, R., Oort, F. \& Bögels, S. (2012). The effectiveness of a school-based mindfulness training as a program to prevent stress in elementary school children. Mindfulness, Online first article, not assigned to an issue. (Preprints), 1-11, November 2012. Available via: http://dx.doi.org/10.1007/s12671012-0171-9.

Winefield, H. (2000). Stress reduction for family caregivers in chronic mental illness: implications of a work stress management perspective. International Journal of Stress Management, 7(3), 193-207.

Zernicke, K., Campbell, T., Blustein, P., Fung, T., Johnson, J., Bacon, S. \& Carlson, L. (2013). Mindfulness-based stress reduction for the treatment of irritable bowel syndrome symptoms: a randomized wait-list controlled trial. International Journal Behavioral Medicine, 20(3), 385-396. Available via: http://dx.doi.org/10.1007/s12529-012-9241-6. 